\title{
Optimization of the $Q$ Factor in Photonic Crystal Microcavities
}

\author{
Jelena Vučković, Marko Lončar, Hideo Mabuchi, and Axel Scherer
}

\begin{abstract}
We express the quality factor of a mode in terms of the Fourier transforms of its field components and prove that the reduction in radiation loss can be achieved by suppressing the mode's wavevector components within the light cone. Although this is intuitively clear, our analytical proof gives us insight into how to achieve the $Q$ factor optimization, without the mode delocalization. We focus on the dipole defect mode in free-standing membranes and achieve $Q>10^{4}$, while preserving the mode volume of the order of one half of the cubic wavelength of light in the material. The derived expressions and conclusions can be used in the optimization of the $Q$ factor for any type of defect in planar photonic crystals.
\end{abstract}

Index Terms-FDTD methods, Fourier transforms, integrated optics, optical resonators, optics, optoelectronic devices, $Q$ factor.

\section{INTRODUCTION}

$\mathbf{O}$ $\mathrm{NE}$ OF the greatest challenges in photonic crystal research is the construction of optical microcavities with small mode volumes and large quality factors, for efficient localization of light. Beside standard applications of these structures (such as lasers or filters), they can potentially be used for cavity QED experiments, or as building blocks for quantum networks. Although three-dimensional (3-D) photonic crystals offer the opportunity to manipulate light in all three dimensions in space, many research groups have focused their efforts on planar photonic crystals (i.e., two-dimensional (2-D) photonic crystals of finite depth) in recent years [1]-[13]. The fabrication procedures of planar photonic crystals are much simpler than those of their 3-D counterparts, but their light confinement is only "quasi-3D" and resulting from the combined action of the 2-D photonic crystal and internal reflection. The imperfect confinement in the third dimension produces some unwanted out-of-plane loss (radiation loss), which is usually a limiting factor in the performance of these structures. The problem of the $Q$ factor optimization in planar photonic crystal microcavities has been addressed recently by several groups [2], [14]-[17]. The cavities that we proposed have a potential for achieving $Q>10^{4}$ together with the mode volume of the order of one half of the cubic wavelength of light in the material [2], [16]. We have also recently demonstrated an experimental $Q$ factor of 2800 in this type of structure,

Manuscript received September 18, 2001; revised January 8, 2002. This work was supported by the Caltech MURI Center for Quantum Networks.

J. Vučković is with the Edward L. Ginzton Laboratory, Stanford University, Stanford, CA 94305-4085 USA.

M. Lončar, H. Mabuchi, and A. Scherer are with the California Institute of Technology, Pasadena, CA 91125 USA.

Publisher Item Identifier S 0018-9197(02)05687-7.

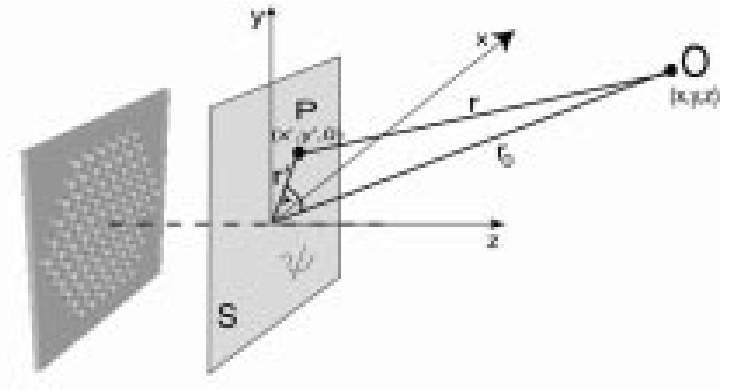

Fig. 1. Estimating the radiation field at the observation point $O$ from the known near field at the surface $S$.

for which the theoretically predicted $Q$ was around 4000 [18]. In our earlier work [2], we have only briefly addressed the mechanism behind the reduction of radiation loss in our structures: the suppression of wavevector components of the defect mode that are positioned within the light cone. In this paper, we discuss this phenomenon in detail and derive the analytical expression relating the $Q$ factor of a mode to the Fourier transform of the mode pattern. We also show how to suppress the wavevector components within the light cone, without delocalizing a mode. Although our study focuses only on the dipole mode, the derived relations are universal and conclusions can be used in the optimization of the $Q$ factor for any type of mode and defect in planar photonic crystals.

\section{RELATION BETWEEN THE $Q$ FACTOR AND THE FOURIER TRANSFORM OF A MODE}

The 3-D finite difference time-domain (FDTD) analysis can provide us with the near-field distribution of the analyzed microcavity. FDTD analysis of the far field would require large amounts of computer memory and would be computationally inefficient. However, we can compute the far field starting from the known near-field distribution. Any wavefront can be considered as a source of secondary waves that add to produce distant wavefronts, according to Huygens principle. Let us assume that we know the field distribution across the surface $S$, positioned in the near field and above the free-standing membrane, as in Fig. 1. Our goal is to estimate the far field at the observation point $O$. The far fields can be considered as arising from the equivalent current sheets at the surface $S$ [19]. For example, let $S$ be the plane positioned at $z=0$, parallel to the surface of the membrane, and at a small distance $\Delta z$ from it. This choice of surface $S$ will allow us to relate the $Q$ factor of a mode to the Fourier transform of its field pattern. The equivalent sources in 
the plane $S$ can be represented in terms of the surface electric $\left(\vec{J}_{s}\right)$ and magnetic $\left(\vec{M}_{s}\right)$ currents

$$
\begin{aligned}
\vec{J}_{s} & =\hat{n} \times \vec{H}=-\hat{x} H_{y}+\hat{y} H_{x} \\
\vec{M}_{s} & =-\hat{n} \times \vec{E}=\hat{x} E_{y}-\hat{y} E_{x}
\end{aligned}
$$

where $\hat{n}$ is a normal to the surface $S$, and $\vec{E}$ and $\vec{H}=\vec{B} / \mu_{0}$ are the electric and magnetic fields, respectively. In a homogeneous, isotropic medium above $S$, a retarded potential $\vec{A}$ and a second retarded potential $\vec{F}$ can be estimated from the previously introduced surface currents

$$
\begin{aligned}
& \vec{A}=\mu_{0} \int_{S} \frac{\vec{J}_{s} e^{-i k r}}{4 \pi r} d S \\
& \vec{F}=\epsilon_{0} \int_{S} \frac{\vec{M}_{s} e^{-i k r}}{4 \pi r} d S
\end{aligned}
$$

where $k$ is defined as $k=2 \pi / \lambda=\omega / c$ ( $\lambda$ is the mode wavelength measured in air) and $r$ is the distance between the point where the potentials are evaluated and the surface element $d S$ (i.e., between the points $O$ and $P$ ).

From Fig. 1, it follows that $r \approx r_{0}-r^{\prime} \cos (\psi)$. Let us now introduce the radiation vectors $\vec{L}$ and $\vec{N}$

$$
\begin{aligned}
\vec{N} & =\int_{S} \vec{J}_{s} e^{i k r^{\prime} \cos (\psi)} d S \\
\vec{L} & =\int_{S} \vec{M}_{s} e^{i k r^{\prime} \cos (\psi)} d S .
\end{aligned}
$$

Then we have

$$
\begin{aligned}
\vec{A} & =\mu_{0} \frac{e^{-i k r_{0}}}{4 \pi r_{0}} \vec{N} \\
\vec{F} & =\epsilon_{0} \frac{e^{-i k r_{0}}}{4 \pi r_{0}} \vec{L} .
\end{aligned}
$$

From Fig. 1, we also have

$$
k r^{\prime} \cos (\psi)=\frac{k}{r_{0}}\left(x x^{\prime}+y y^{\prime}\right)
$$

where $\left(x^{\prime}, y^{\prime}, 0\right)$ are the coordinates of the point $P$ in the plane $S$, and $(x, y, z)$ are the coordinates of the observation point $O$.

From (5) and (6), it follows that radiation vectors $\vec{N}$ and $\vec{L}$ represent the 2-D Fourier transforms of the surface currents $\vec{J}_{s}$ and $\vec{M}_{s}$, evaluated at the value of the wavevector $\vec{k}_{\|}=k\left(x / r_{o} \hat{x}+y / r_{o} \hat{y}\right)$ (in rectangular coordinates), i.e., $\vec{k}_{\|}=k \sin \theta(\cos \phi \hat{x}+\sin \phi \hat{y})$ in circular polar coordinates

$$
\begin{aligned}
\vec{N} & =\left.F T_{2}\left(\vec{J}_{s}\right)\right|_{\vec{k}_{\|}=k\left(x / r_{0}, y / r_{0}\right)} \\
\vec{L} & =\left.F T_{2}\left(\vec{M}_{s}\right)\right|_{\vec{k}_{\|}=k\left(x / r_{0}, y / r_{0}\right)} \\
F T_{2}(f(x, y)) & =\iint d_{x} d_{y} f(x, y) e^{i \vec{k}_{\|} \cdot(x, y)} \\
& =\iint d_{x} d_{y} f(x, y) e^{i\left(k_{x} x+k_{y} y\right)}
\end{aligned}
$$

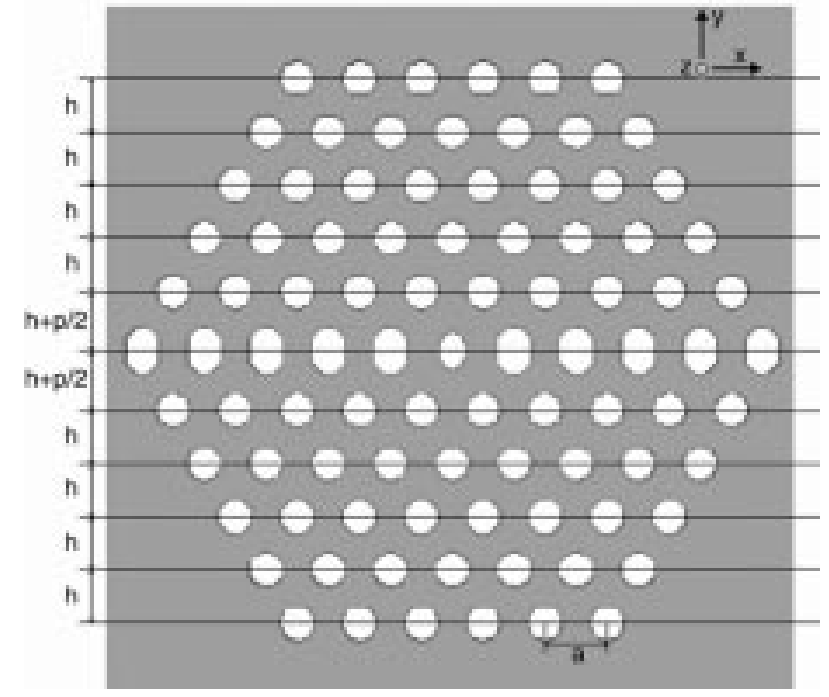

Fig. 2. Microcavity structure consisting of a single defect (produced by reducing the radius of the central hole to $r_{\text {def }} / a=0.2$ from $r / a=0.275$ ) and a fractional edge dislocation of order $p=4$ along the $x$ axis. The applied discretization is 20 pixels per periodicity $a$.

Components of radiation vectors can, therefore, be expressed in terms of the Fourier transforms of the field components at the surface $S$

$$
\begin{aligned}
N_{x} & =-\left.F T_{2}\left(H_{y}\right)\right|_{\vec{k}_{\|}} \\
N_{y} & =\left.F T_{2}\left(H_{x}\right)\right|_{\vec{k}_{\|}} \\
L_{x} & =\left.F T_{2}\left(E_{y}\right)\right|_{\vec{k}_{\|\|}} \\
L_{y} & =-\left.F T_{2}\left(E_{x}\right)\right|_{\vec{k}_{\|}} \\
\vec{k}_{\|} & =k\left(\frac{x}{r_{0}}, \frac{y}{r_{0}}\right)=k \sin \theta(\cos \phi \hat{x}+\sin \phi \hat{y}) .
\end{aligned}
$$

It is important to note that, for any observation point $O$, the previously introduced wavevector $\vec{k}_{\|}$lies within the light cone (i.e., $\left|\vec{k}_{\|}\right| \leq k$, where $k=2 \pi / \lambda$ ). Therefore, radiation vectors are purely determined by Fourier components located within the light cone.

Far fields can be expressed in terms of retarded potentials as

$$
\begin{aligned}
& \vec{E}=-i \omega \vec{A}-\frac{i \omega}{k^{2}} \nabla(\nabla \cdot \vec{A})-\frac{1}{\epsilon_{0}} \nabla \times \vec{F} \\
& \vec{H}=-i \omega \vec{F}-\frac{i \omega}{k^{2}} \nabla(\nabla \cdot \vec{F})-\frac{1}{\mu_{0}} \nabla \times \vec{A} .
\end{aligned}
$$

Under the assumption that all terms in fields decaying faster than $1 / r_{o}$ can be neglected, the electric field components at an arbitrary point $O$ are

$$
\begin{aligned}
E_{\theta} & =\eta H_{\phi}=-i \frac{e^{-i k r_{o}}}{2 \lambda r_{o}}\left(\eta N_{\theta}+L_{\phi}\right) \\
E_{\phi} & =-\eta H_{\theta}=i \frac{e^{-i k r_{o}}}{2 \lambda r_{o}}\left(-\eta N_{\phi}+L_{\theta}\right) \\
\eta & =\sqrt{\frac{\mu_{o}}{\epsilon_{o}}}
\end{aligned}
$$



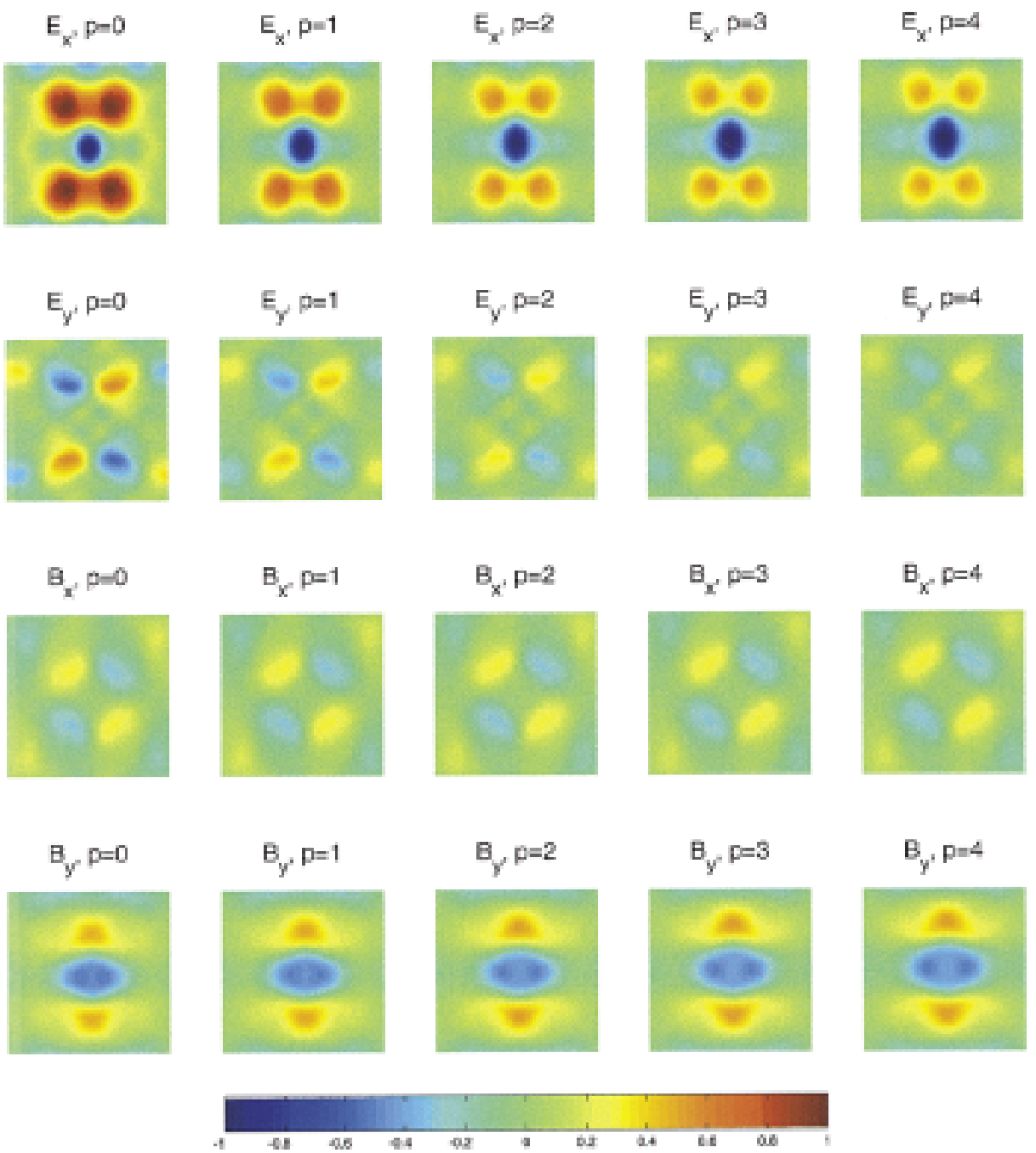

Fig. 3. Field components of the $x$-dipole mode at the surface $S$ positioned at approximately $d / 4$ from the surface of the membrane. The analyzed structure is shown in Fig. 2.

where $\left(r_{o}, \theta, \phi\right)$ represent the coordinates of the point $O$ in the spherical polar coordinate system. The radiation intensity (power per unit solid angle) is then equal to [19]

$$
K(\theta, \phi)=\frac{\eta}{8 \lambda^{2}}\left(\left|N_{\theta}+\frac{L_{\phi}}{\eta}\right|^{2}+\left|N_{\phi}-\frac{L_{\theta}}{\eta}\right|^{2}\right)
$$

and the total averaged power radiated into the half-space $z>0$ is given by

$$
P_{1}=\int_{0}^{\pi / 2} \int_{0}^{2 \pi} d_{\theta} d_{\phi} \sin (\theta) K(\theta, \phi) .
$$

The radiation vectors in spherical polar coordinates can be expressed from their components in rectangular coordinates

$$
\begin{aligned}
& N_{\theta}=\left(N_{x} \cos \phi+N_{y} \sin \phi\right) \cos \theta \\
& N_{\phi}=-N_{x} \sin \phi+N_{y} \cos \phi
\end{aligned}
$$

where $N_{x}, N_{y}, L_{x}$, and $L_{y}$ were previously given as the 2-D Fourier transforms of the appropriate field components tangential to the surface $S$. Hence, just by knowing the Fourier trans- forms of the tangential field components at the plane $S$, we can evaluate the total averaged power radiated and the far-field distribution. Furthermore, the radiated power depends only on the wavevector components located within the light cone. Therefore, the reduction in radiation loss and the improvement in the $Q$ factor can be achieved by suppressing the Fourier components within the light cone or by redistributing them outside the light cone.

In the case when most of the radiated power is collected at vertical incidence (i.e., at small $\theta$ ), (24) can be simplified as follows:

$$
\begin{aligned}
P_{2}= & \frac{\eta}{8 \lambda^{2} k^{2}} \iint_{\left|\vec{k}_{\|}\right| \leq k} d \vec{k}\left(\left|N_{x}+\frac{L_{y}}{\eta}\right|^{2}+\left|N_{y}-\frac{L_{x}}{\eta}\right|^{2}\right) \\
= & \frac{\eta}{8 \lambda^{2} k^{2}} \iint_{\left|\vec{k}_{\|}\right| \leq k} d \vec{k}\left(\left|F T_{2}\left(H_{y}\right)+\frac{1}{\eta} F T_{2}\left(E_{x}\right)\right|^{2}\right. \\
& \left.+\left|F T_{2}\left(H_{x}\right)-\frac{1}{\eta} F T_{2}\left(E_{y}\right)\right|^{2}\right) .
\end{aligned}
$$


The integral of the cross terms in (27) gives approximately one half of the radiated power. This can be proved easily by starting from the expansion of fields in terms of the Fourier components and the expression for the radiated power as the integral of the $z$ component of the Poynting vector $\vec{\Gamma}$ over the surface $S$. This leads to the following expression for the averaged radiated power:

$$
\begin{array}{r}
P_{3}=2 \frac{\eta}{8 \lambda^{2} k^{2}} \iint_{\left|\overrightarrow{\vec{k}_{\|} \mid}\right| \leq k} d \vec{k}\left(\left|F T_{2}\left(H_{x}\right)\right|^{2}+\left|F T_{2}\left(H_{y}\right)\right|^{2}\right. \\
\left.+\frac{1}{\eta^{2}}\left|F T_{2}\left(E_{x}\right)\right|^{2}+\frac{1}{\eta^{2}}\left|F T_{2}\left(E_{y}\right)\right|^{2}\right) .
\end{array}
$$

It is important to note that, if some field component $u(x, y)$ is odd with respect to the $x$ coordinate [i.e., $u(x, y)=-u(-x, y)]$, then its Fourier transform must be equal to zero for any point in the Fourier space with $k_{x}=0$. Similarly, any field component which is odd with respect to the $y$ coordinate has a Fourier transform which is zero for any point with $k_{y}=0$.

Let us introduce the radiation factor $R F$ which is directly proportional to the radiated power $P$

$$
R F_{i}=\frac{P_{i}}{W}, \quad i=1,2,3, \ldots
$$

where $W$ represents the total energy of a mode in the half-space above the middle of the membrane. The radiation $Q$ factor of a mode (which is a measure of the radiation, out-of-plane loss) can be expressed as

$$
Q=\omega \frac{W}{P}=\frac{\omega}{R F}
$$

\section{EFFECt OF Fractional Edge Dislocations ON THE $Q$ FACTOR OF THE DIPOLE MODE IN FREE-STANDING MEMBRANES}

We have recently proposed the design and fabrication of optical microcavities in free-standing membranes with $Q>10^{4}$ for the dipole mode, and mode volumes of the order of one half of the cubic wavelength of light (measured in the material) [2], [16]. The dramatic improvement in $Q$ factors over single defect microcavities (without a significant increase in the mode volume) was obtained by introducing a novel type of photonic crystal lattice defect, consisting of the elongation of holes along the symmetry axes. We call this type of defect a fractional edge dislocation, by analogy with edge dislocations in solid state physics. Edge dislocations are formed by introducing extra atomic planes into the crystal lattice. On the other hand, we insert here only fractions of the atomic planes along the symmetry axes of the photonic crystal, as shown in Fig. 2. Hole-to-hole distances are preserved under this deformation, and the half-spaces $y>p / 2$ and $y<-p / 2$ maintain the unperturbed photonic crystal geometry.

We consider again some of the microcavities that we proposed in [2]. The unperturbed photonic crystal parameters are $r / a=0.275, d / a=0.75$, and $n=3.4$, where $r, a, d$, and $n$ represent the hole radius, the periodicity of the triangular lattice,
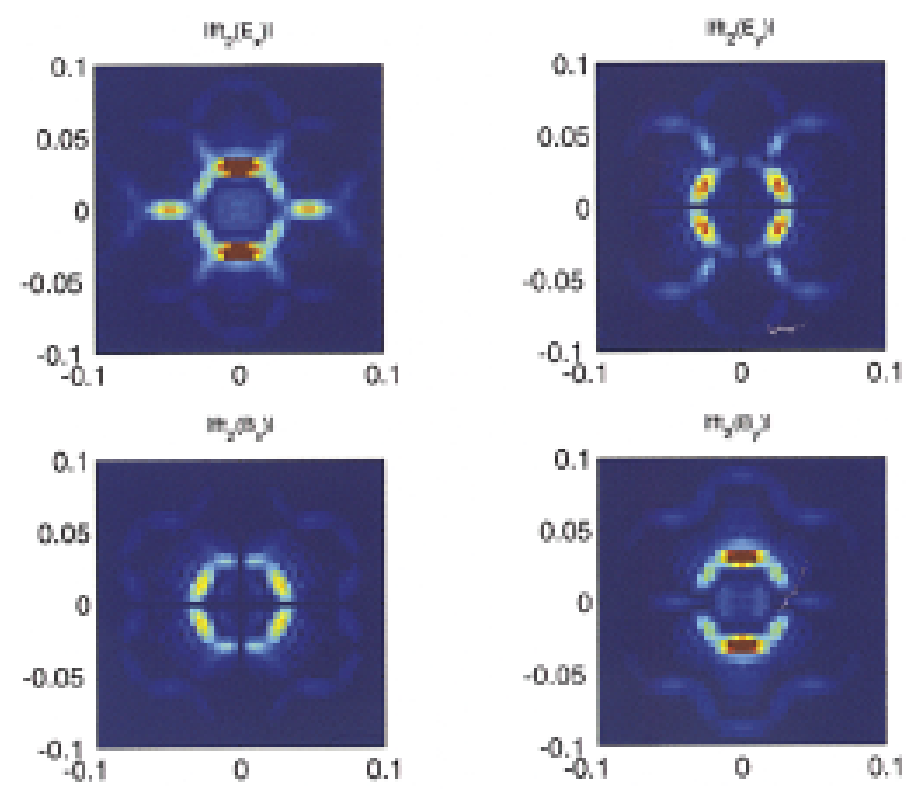

Fig. 4. Fourier components of the $x$-dipole mode in the structure from Fig. 2. A fractional edge dislocation is of the order $p=0$ in this case. The light cone can be represented as a disk with the radius approximately equal to 0.015 located in the center of each square. The horizontal and vertical axes correspond to $k_{2} / 2 \pi$ and $k_{y} / 2 \pi$, respectively.

the thickness of the slab, and the refractive index of the semiconductor material, respectively. The choice of photonic crystal parameters is discussed in more detail in our previous work [2], [20]. Briefly, we limit the $r / a$ ratio to rather modest values of around 0.3 , in order to minimize the out-of-plane losses produced by the vertical scattering at the edges of holes. Since the reduction in $r / a$ leads to a decrease in the size of the bandgap, it is important not to reduce the hole radius too much, in order to preserve the lateral confinement and small mode volume (e.g., we do not use $r / a$ below 0.275 ). The $d / a$ ratio of our structures is usually between 0.65 and 0.75 , and we were able to design microcavities with very high $Q$ factors at both ends of this range, without a significant change in the mode volume [2], [20]. The reasons for choosing this thickness range are the following: if the slab is too thin, the mode is not confined well within it vertically, and it interacts more strongly with the substrate (positioned at around $\lambda / 2$ underneath the bottom membrane surface in our structures [2]), which reduces its $Q$ factor. Furthermore, the fabrication of thin suspended membranes is difficult, and these structures are not robust. On the other hand, if $d / a$ is too large, the structure is multimode in the vertical direction, which is also undesirable. In the FDTD method, we apply the discretization of 20 pixels per periodicity $a$. Therefore, a fractional edge dislocation of order $p=1$ corresponds to the insertion of extra material whose thickness is equal to $1 / 20 \mathrm{a}$. In the microcavity of our interest, the central hole radius is decreased to $r_{\text {def }} / a=0.2$ and a fractional edge dislocation of order $p$ is applied along the $x$ axis, as shown in Fig. 2. The dipole mode's frequency decreases as a function of the elongation parameter $p$ [2], [20], and it is desirable to start in the elongation process with a mode whose frequency is close to the edge of the air band, allowing enough space to achieve the optimum $Q_{\perp}$ within the bandgap when the structure is tuned. In that case, the lateral 


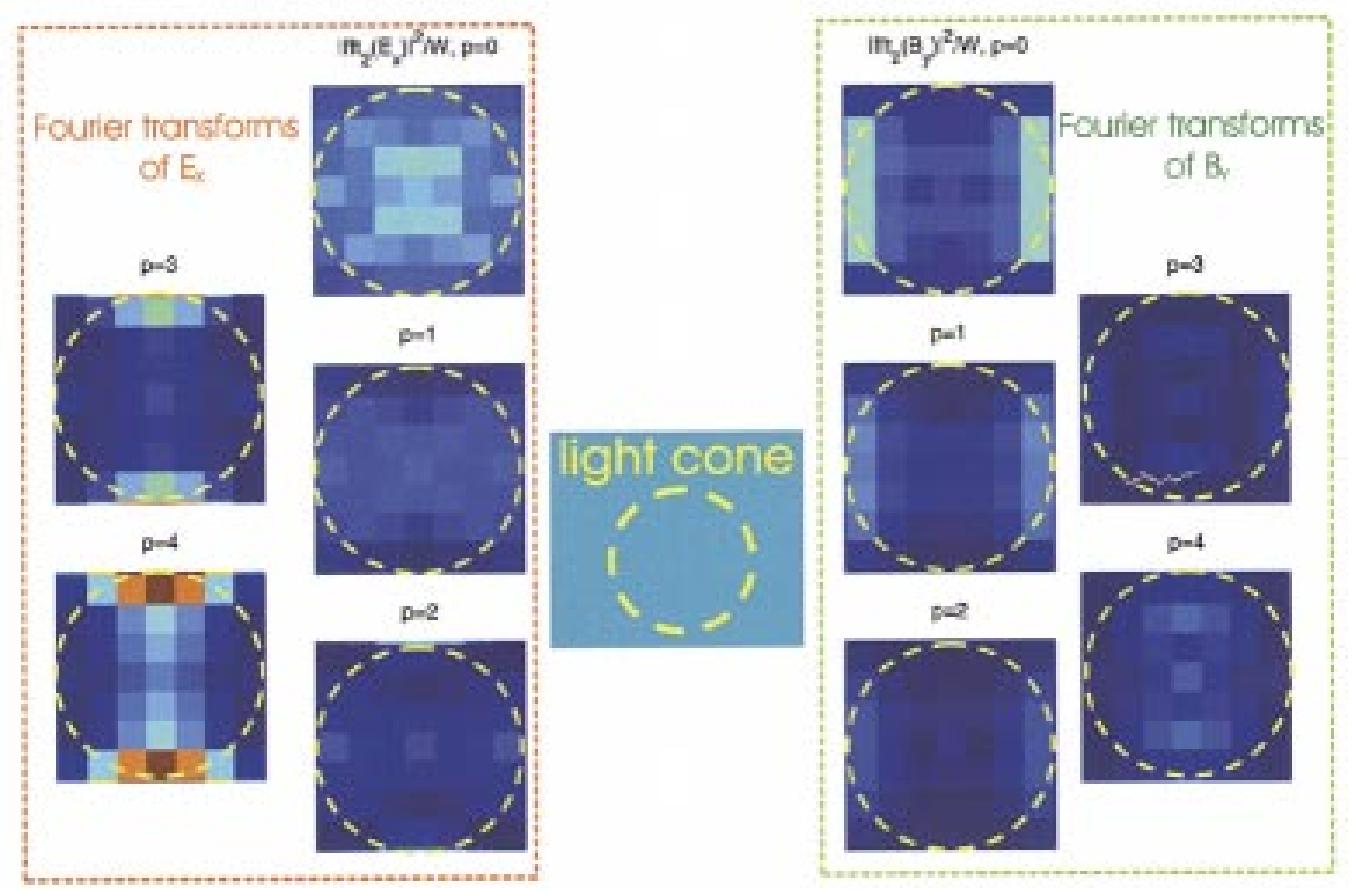

Fig. 5. The 2-D Fourier transforms of the even field components of the $x$-dipole mode in the structure shown in Fig. 2, as a function of the elongation parameter $p$. The light cone can be represented as a disk inscribed into the square. Clearly, the intensities of the Fourier transforms within the light cone are minimized for $p=2$, where the $Q$ factor reaches its maximum.

confinement is preserved and $Q_{\|}$can be improved by increasing the number of photonic crystal layers around the defect. This is one of the reasons for reducing the defect hole radius to only $0.2 a$. The other reason is our long-term goal, a photonic crystal cavity QED with neutral atoms [2], for which we need a strong field intensity within an air hole large enough to place a neutral atom, without significant surface effects. Field components of the $x$-dipole mode in the analyzed structure are shown in Fig. 3, as a function of the elongation parameter $p$. For the $x$-dipole mode, the $E_{x}$ and $B_{y}$ components are even, while the $E_{y}$ and $B_{x}$ components are odd with respect to both symmetry axes $x$ and $y$. Therefore, it is expected that $E_{y}$ and $B_{x}$ (i.e., $L_{x}$ and $N_{y}$ ) do not contribute significantly to the radiated power in this case, since their Fourier transforms are equal to zero along both the $k_{x}$ and $k_{y}$ axes. This is also illustrated in Fig. 4. Therefore, in the case of the analyzed $x$-dipole mode we can approximate the expression (28) even further as

$$
\begin{array}{r}
P_{4}=2 \frac{\eta}{8 \lambda^{2} k^{2}} \iint_{\left|\vec{k}_{\|}\right| \leq k} d_{k_{x}} d_{k_{y}} \cdot\left(\left|F T_{2}\left(H_{y}\right)\right|^{2}\right. \\
\left.+\frac{1}{\eta^{2}}\left|F T_{2}\left(E_{x}\right)\right|^{2}\right) .
\end{array}
$$

In order to minimize the radiated power, it is necessary to minimize (within the light cone) the Fourier transforms of the even field components $E_{x}$ and $B_{y}$. In the general case, these Fourier transforms are nonzero at small values of $\left|\vec{k}_{\|}\right|$(i.e., in the light cone). However, they can be minimized by balancing the intensities of positive and negative field lobes. Indeed, we can observe in Fig. 3 that, by varying the elongation parameter $p$, we also tune the sizes of the central (negative) lobes in $E_{x}$ and $B_{y}$, as well as the intensity distribution between the positive and negative lobes. Therefore, the tuning in $p$ is expected to lead to tuning in the Fourier transforms of the even field components, and subsequently to tuning in radiated powers.

The Fourier components of the $x$-dipole mode in the structure with $p=0$ are shown in Fig. 4. When the elongation parameter $p$ changes in the analyzed range from 0 to 4 , peaks in the Fourier space preserve their position, but their intensities are tuned. This can be observed in Fig. 5. Clearly, Fourier components within the light cone are minimized for $p=2$, where the $Q$ factor reaches its maximum. Therefore, the optimization of the $Q$ factor of the dipole mode (after the application of fractional edge dislocations) is a result of suppression of the wavevector components within the light cone. This suppression is a product of balancing between the energies of the positive and negative field lobes of the even field components. The balancing is obtained by tuning the sizes of the negative lobes with insertion of extra material along the symmetry axis. The $Q$ factor optimization is achieved in this case without a significant mode delocalization.

In our FDTD calculations [2], the total $Q$ factor is separated into the lateral $\left(Q_{\|}\right)$and vertical $\left(Q_{\perp}\right)$ quality factors. $Q_{\perp}$ is a measure of radiation loss, while $Q_{\|}$corresponds to the loss through the mirrors in the lateral directions, which can be reduced by adding more layers of photonic crystal. The boundary for separation of vertical from lateral loss (i.e., $Q_{\perp}$ from $Q_{\|}$) is positioned approximately at $\lambda / 2$ from the surface of the membrane, as suggested in our early work [21]. We have discussed in our recent publication [2] that this choice of boundary excludes some small portion of radiation loss from $Q_{\perp}$, and the total $Q$ factor of the analyzed dipole mode achievable by increasing the 


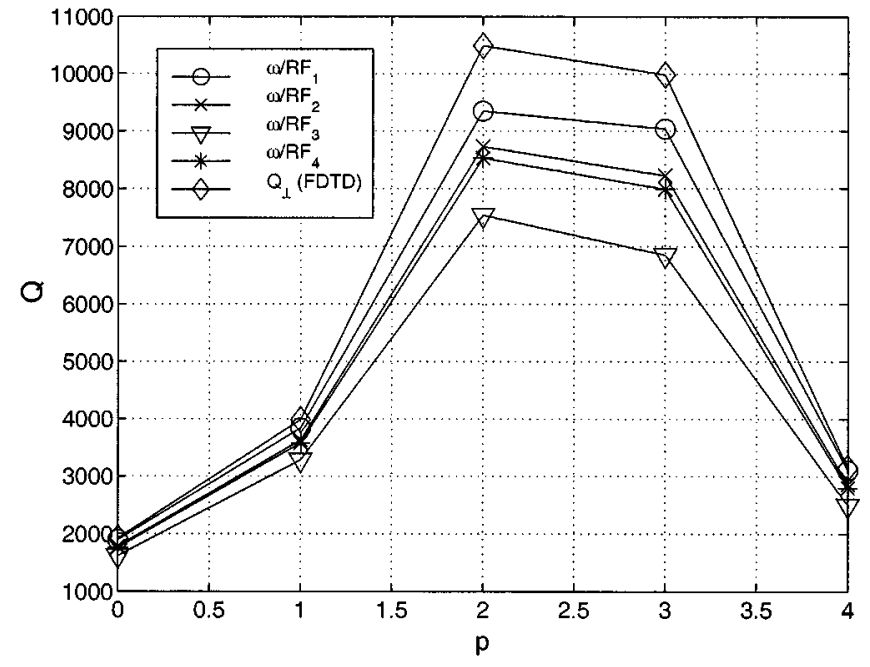

(a)

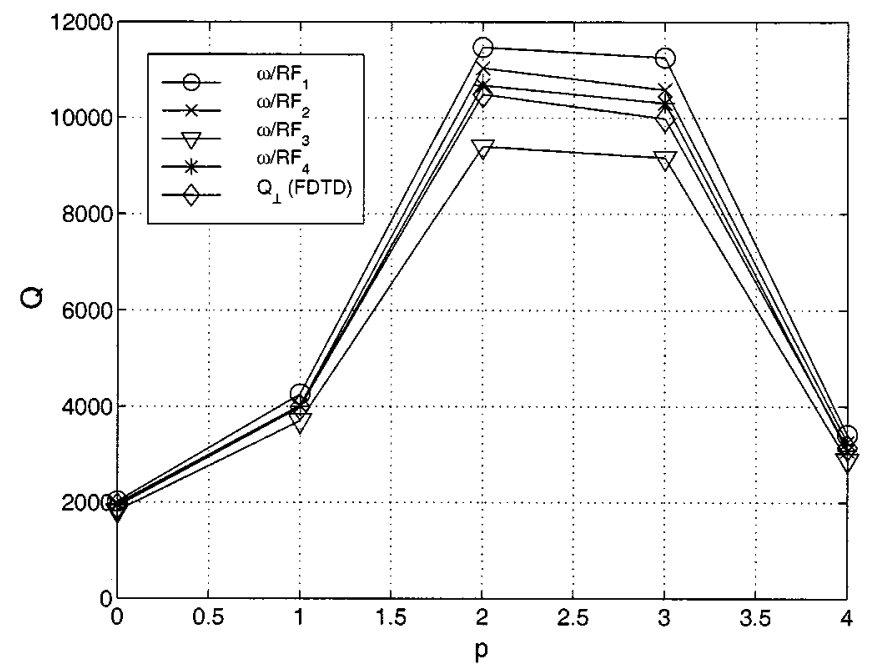

(b)

Fig. 6. $Q$ factors estimated from the FDTD, or from the Fourier transforms of the tangential field components. The plane $S$ is positioned (a) directly above the surface of the membrane, at a distance equal to $d / 4$ from it, and (b) at a distance equal to $\lambda / 2$ from the surface of the membrane.

number of the PC layers around the defect (also referred to as the limit of total $Q$ factor) is somewhat smaller than $Q_{\perp}$. For this reason, we now believe that a better choice of boundary for separation of $Q_{\perp}$ from $Q_{\|}$would be the one positioned directly above the surface of the membrane. However, for the purpose of comparing our new results [2] with our earlier work [21], we preserve this boundary positioned at $\lambda / 2$ from the surface of the membrane.

The radiation $Q$ factors are evaluated using the method presented in this paper, and results are shown in Fig. 6. The plane $S$ (above which we integrate the radiated power) is positioned directly above and at $\lambda / 2$ above the surface of the membrane, in Fig. 6(a) and (b), respectively. Therefore, in the latter case, we expect a better agreement between the radiation $Q$ factors $(\omega / R F)$ estimated using our new method and $Q_{\perp}$ estimated from the FDTD calculations, but in the former case the newly calculated $Q$ factors are a better approximation of the limit of the total $Q$ factors. In Fig. 6(a), $Q_{\perp}$ factors are larger than $Q$ factors calculated as $\omega / R F$, because $\omega / R F$ includes practically all ra- diation (out-of-plane) loss, while $Q_{\perp}$ includes only the radiation loss above $\lambda / 2$ from the surface of the membrane. In Fig. 6(b), both the plane $S$ and the boundary for separation of $Q_{\perp}$ from $Q_{\|}$ are positioned at $\lambda / 2$ from the membrane surface, and a better agreement with $Q_{\perp}$ from the FDTD simulations is observed. However, $Q_{\perp}$ is somewhat smaller, due to numerical inaccuracy. Radiation factors $R F_{2}, R F_{3}$, and $R F_{4}$ are estimated under the assumption that most of the radiation is collected at vertical incidence. This is not really true in the case of the $x$-dipole, for which reason there is an offset between the $Q$ factors evaluated from $R F_{i}, i=2,3,4$, and $Q$ estimated from $R F_{1}$, which does not make any assumptions regarding the direction of radiation.

\section{CONCLUSION}

We have presented a method for estimating the $Q$ factor of a mode and its radiation loss from the known Fourier transform of the near-field distribution. By applying this approach to high $Q$ structures that we have proposed recently [2], we have proven that the optimization of the $Q$ factor of the dipole defect mode (after the application of fractional edge dislocations) results from the suppression of the wavevector components within the light cone. This suppression is a result of balancing between the positive and negative lobes in the even field components. The balancing is obtained by tuning the sizes of the negative lobes with insertion of extra material along the symmetry axis. Although our analysis focuses on the dipole mode only, a similar approach can be applied to any type of microcavity formed in planar photonic crystals.

\section{ACKNOWLEDGMENT}

One of the authors, J. Vučković, would like to thank Prof. Y. Yamamoto, Stanford University, Stanford, CA, for many fruitful discussions.

\section{REFERENCES}

[1] M. Lončar, D. Nedeljković, T. Doll, J. Vučković, A. Scherer, and T. P. Pearsall, "Waveguiding at $1500 \mathrm{~nm}$ using photonic crystal structures in silicon on insulator wafers," Appl. Phys. Lett., vol. 77, pp. 1937-1939, Sept. 2000.

[2] J. Vučković, M. Lončar, H. Mabuchi, and A. Scherer, "Design of photonic crystal microcavities for cavity QED," Phys. Rev. E, vol. 65, no. 016608, Jan. 2001.

[3] O. Painter, R. Lee, A. Scherer, A. Yariv, J. O'Brien, P. Dapkus, and I. Kim, "Two-dimensional photonic band-gap defect mode laser," Science, vol. 284, pp. 1819-1821, June 1999.

[4] T. Yoshie, A. Scherer, H. Chen, D. Huffaker, and D. Deppe, "Optical characterization of two-dimensional photonic crystal cavities with indium arsenide quantum dot emitters," Appl. Phys. Lett., vol. 79, no. 1, pp. 114-116, July 2001.

[5] P. Villeneuve, S. Fan, S. Johnson, and J. Joannopoulos, "Three dimensional photon confinement in photonic crystals of low-dimensional periodicity," Proc. Inst. Elect. Eng., pt. J, vol. 145, pp. 384-390, Dec. 1998.

[6] D. Labilloy, H. Benisty, C. Weisbuch, C. Smith, T. Krauss, R. Houdre, and U. Oesterle, "Finely resolved transmission spectra and band structure of two-dimensional photonic crystals using emission from InAs quantum dots," Phys. Rev. B, vol. 59, pp. 1649-1652, Jan. 1999.

[7] S. Noda, A. Chutinan, and M. Imada, "Trapping and emission of photons by a single defect in a photonic bandgap structure," Nature, vol. 407, no. 6804, pp. 608-610, Oct. 2000.

[8] M. Boroditsky, R. Vrijen, T. Krauss, R. Coccioli, R. Bhat, and E. Yablonovitch, "Spontaneous emission extraction and Purcell enhancement from thin-film 2-D photonic crystals," J. Lightwave Technol., vol. 17, pp. 2096-2112, Nov. 1999. 
[9] J. K. Hwang, H. Y. Ryu, D. S. Song, I. Y. Han, H. W. Song, H. K. Park, Y. H. Lee, and D. H. Jang, "Room-temperature triangular-lattice twodimensional photonic band gap lasers operating at $1.54 \mu \mathrm{m}$," Appl. Phys. Lett., vol. 76, no. 21, pp. 2982-2984, May 2000.

[10] T. Baba, N. Fukaya, and A. Motegi, "Clear correspondence between theoretical and experimental light propagation characteristics in photonic crystal waveguides," Electron. Lett., vol. 37, no. 12, pp. 761-762, June 2001.

[11] C. J. M. Smith, T. F. Krauss, H. Benisty, M. Rattier, C. Weisbuch, U. Oesterle, and R. Houdre, "Clear correspondence between theoretical and experimental light propagation characteristics in photonic crystal waveguides," J. Opt. Soc. Amer. B, vol. 17, no. 12, pp. 2043-2051, Dec. 2000.

[12] C. Monat, C. Seassal, X. Letartre, P. Viktorovitch, P. Regreny, M. Gendry, P. Rojo-Romeo, G. Hollinger, E. Jalaguier, S. Pocas, and B. Asper, "InP 2D photonic crystal microlasers on silicon wafer: Room temperature operation at $1.55 \mu \mathrm{m}$," Electron. Lett., vol. 37, no. 12, pp. 764-766, June 2001

[13] N. Kawai, K. Inoue, N. Carlsson, N. Ikeda, Y. Sugimoto, K. Asakawa, and T. Takemori, "Confined band gap in an air-bridge type of two-dimensional AlGaAs photonic crystal," Phys. Rev. Lett., vol. 86, no. 11, pp. 2289-2292, Mar. 2001.

[14] S. G. Johnson, S. Fan, A. Mekis, and J. D. Joannopoulos, "Multipolecancellation mechanism for high-Q cavities in the absence of a complete photonic band gap," Appl. Phys. Lett., vol. 78, no. 22, pp. 3388-3390, May 2001.

[15] E. Miyai and and K. Sakoda, "Quality factor for localized defect modes in a photonic crystal slab upon a low-index dielectric substrate," Opt. Lett., vol. 26, no. 10, pp. 740-742, May 2001.

[16] J. Vučković, M. Lončar, H. Mabuchi, and A. Scherer, "Photonic crystal microcavities for strong coupling between an atom and the cavity field," in Proc. LEOS 2000, Rio Grande, Puerto Rico, Nov. 2000.

[17] H. Y. Ryu, S. H. Kim, H. G. Park, J. K. Hwang, and Y. H. Lee, "Squarelattice photonic gand-gap sincle-cell laser operating in the lowest-order whispering gallery mode,", vol. 80, no. 21, pp. 3883-3885, May, 2002.

[18] T. Yoshie, J. Vučković, A. Scherer, H. Chen, and D. Deppe, "High quality two-dimensional photonic crystal slab cavities," Appl. Phys. Lett., vol. 79, no. 26, pp. 4289-4291, Dec. 2001.

[19] S. Ramo, J. R. Whinnery, and T. Van Duzer, Fields and Waves in Communication Electronics. New York: Wiley, 1994.

[20] J. Vučković, "Photonic crystal structures for efficient localization or extraction of light," Ph.D. dissertation, California Inst. of Technology, Pasadena, 2002.

[21] O. Painter, J. Vučković, and A. Scherer, "Defect modes of a two-dimensional photonic crystal in an optically thin dielectric slab," J. Opt. Soc. Amer. B, vol. 16, no. 2, pp. 275-285, Feb. 1999.
Jelena Vučković received the Ph.D. degree in electrical engineering from the California Institute of Technology (Caltech), Pasadena, in 2002, for her work on photonic crystal-based optical and quantum optical devices.

She is presently a post-doctoral scholar in the Mesoscopic Quantum Optics Group at Stanford University, Stanford, CA, working on a single photon source based on a single quantum dot coupled to a micropost microcavity. Her research interests include photonic crystals, semiconductor cavity QED, quantum optics, and nanofabrication techniques.

Marko Lončar was born in Dubrovnik, Croatia, in 1974. He received the Diploma in engineering from the University of Belgrade, Yugoslavia, in 1997 and the M.S. degree in electrical engineering from California Institute of Technology (Caltech), Pasadena, in 1998. He is currently working toward the $\mathrm{Ph} . \mathrm{D}$. degree in electrical engineering at Caltech.

His research interests include design and fabrication of nano-optics devices and ultrasmall device processing techniques.

Hideo Mabuchi received the A.B. degree from Princeton University, Princeton, $\mathrm{NJ}$, in 1992 and the $\mathrm{Ph} . \mathrm{D}$. degree in physics from the California Institute of Technology (Caltech), Pasadena, in 1998.

He has been on the Caltech faculty since 1998, currently as Associate Professor of Physics. His experimental and theoretical research span a range of topics from quantum measurement and quantum feedback to molecular biophysics.

Dr. Mabuchi is an Office of Naval Research Young Investigator and a John D. and Catherine T. MacArthur Foundation Fellow.

Axel Scherer received the B.S., M.S., and Ph.D. degrees from the New Mexico Institute of Mining and Technology in 1981, 1982 and 1985, respectively.

From 1985 until 1993 he worked in the Quantum Device Fabrication Group at Bellcore. Currently he is the Bernard E. Neches Professor of Electrical Engineering, Applied Physics, and Physics at the California Institute of Technology, Pasadena, specializing in device microfabrication. His research interests include design and fabrication of functional photonic, nanomagnetic, and microfluidic devices. 